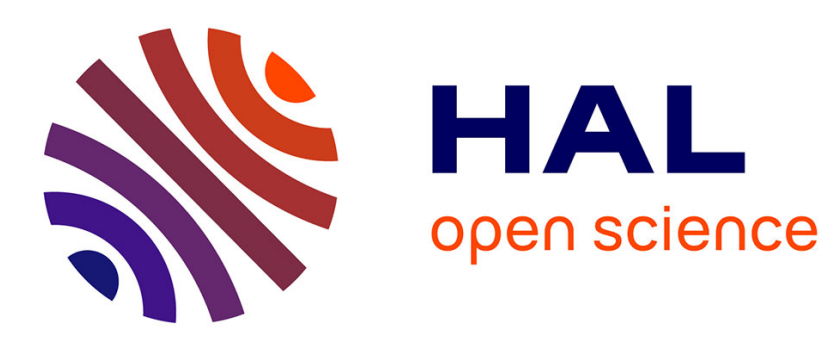

\title{
Introduction of X-ray CT application in geotechnical engineering - theory and practice
}

J Otani, Y Watanabe, Bastien Chevalier

\section{To cite this version:}

J Otani, Y Watanabe, Bastien Chevalier. Introduction of X-ray CT application in geotechnical engineering - theory and practice. 9th World Congress on Computational Mechanics and 4th Asian Pacific Congress on Computational Mechanics, Jul 2010, Sydney, Australia. pp.012089, 10.1088/1757899X/10/1/012089 . hal-02118862

\section{HAL Id: hal-02118862 \\ https://hal.uca.fr/hal-02118862}

Submitted on 17 Nov 2020

HAL is a multi-disciplinary open access archive for the deposit and dissemination of scientific research documents, whether they are published or not. The documents may come from teaching and research institutions in France or abroad, or from public or private research centers.
L'archive ouverte pluridisciplinaire HAL, est destinée au dépôt et à la diffusion de documents scientifiques de niveau recherche, publiés ou non, émanant des établissements d'enseignement et de recherche français ou étrangers, des laboratoires publics ou privés. 


\title{
Introduction of X-ray CT application in geotechnical engineering - theory and practice
}

\author{
J Otani ${ }^{1}$, Y Watanabe and B Chevalier \\ $X$-Earth Center, Graduate School of Science and Technology, Kumamoto University, \\ Kumamoto 860-8555, Japan \\ E-mail: junotani@kumamoto-u.ac.jp
}

\begin{abstract}
In this paper, fundamental issues on the use of X-ray CT to geomaterials were discussed, which were the contents of what you have to know about the use of X-ray CT, especially for geomaterials or granular materials. Then, two of our recent studies were introduced, which are 1) the use of X-ray CT data to quantitative measurements such as deformation and strains and 2) comparison of X-ray CT results with numerical analysis. Here, the triaxial compression test on sand was conducted and the image analysis technique called "Digital Image Correlation" was used to measure displacement and strain fields in the soils using a large number of CT images. For the second topic, one of the advantages on the use of $\mathrm{X}$-ray CT is to investigate the behavior in three dimensions. Here, so called trap door test was conducted with the materials of glass beads and the shape of the square door. DEM analysis was also conducted for the simulation of this behaviour and both results were compared. Based on all the results in this paper, the effectiveness of the use of X-ray CT in geomechanics and geotechnical engineering was revealed.
\end{abstract}

\section{Introduction}

An X-ray CT was developed as a medical diagnosis and recently, this apparatus has been widely used in engineering purposes. The authors started to use so called "Industrial X-ray CT scanner" from the year of 1996 on the topics of soil mechanics and geotechnical engineering, and so far, a large number of research outcomes were produced and those are related to the characterization of the fundamental behavior of soils and the investigation of geotechnical structures (for example, [1],[2], and [3]). Here in this paper, first of all, the fundamental issues on the use of X-ray CT to geomaterials are discussed which are the contents of what you have to know about the use of X-ray CT, especially for geomaterials or granular materials. This part of contents is related to some X-ray Physics with geomaterial properties. Then, two of our recent studies which are 1) the use of X-ray CT data to quantitative measurements such as deformation and strains and 2) comparison of X-ray CT results with numerical analysis are introduced. Finally, based on all the results shown, the usefulness of X-ray $\mathrm{CT}$ in the field of geomechanics and geotechnical engineering is confirmed.

\footnotetext{
${ }^{1}$ To whom any correspondence should be addressed.
} 


\section{Fundamental issues on X-ray CT application to geomaterials [4]}

\subsection{X-ray CT method}

$\mathrm{X}$-ray Computed Tomography (CT) is a non destructive technique that allows imaging and quantification of internal features of an object in three dimensions. The method reveals differences in density and atomic composition. The principle of the measurement consists in two steps: first, recording x-ray radiographies of a specimen at several different angular positions with a full angle of at least $180^{\circ}$ and then, reconstructing virtual slices from these different projections, using appropriate algorithms which are either algebraic or based on a back projection principle. Stacking several sequential slices allows of reconstructing a three-dimensional image of the object, in which one of the examples is shown in Fig. 1 for the case of soils. CT images are constructed by the spatial distribution of so called "CT-value" and this is defined as:

$$
\text { CT-value }=\frac{\mu_{t}-\mu_{w}}{\mu_{w}} K
$$

where $\mu_{t}:$ coefficient of absorption at scanning point, $\mu_{w}:$ coefficient of absorption for water, and $K$ : constant (Hounsfield value). Here, it is noted that this constant is fixed to a value of 1000 . Thus, the CT-value of air should be -1000 because the coefficient of absorption for air is zero. Likewise, CTvalue for water is 0 from the definition of Eq. (1). CT images are presented with shaded gray or black color for low CT-value and light gray or white color for high CT-value in all the subsequent black and white colors. The total number of levels on these colors is 256. It is well known that this CT-value is linearly related to the material density. Fig. 2 shows a relationship between CT-value and dry density of sand. From this figure, although CT-value is affected by both density of the materials and its atomic composition, it is confirmed that there is a linear relationship between CT-value and the density of the material. And this relationship depends on the scanning conditions such as x-ray attenuation, and the size and shape of the scanning materials. Therefore, the size and shape of the scanning materials have to be fixed for all the comparative studies and the conditions of x-ray attenuation is discussed next.

\subsection{CT scanning}

There are some numbers of scanning conditions to be determined properly and this is totally depending on the material property to be scanned. A determination of voltage for $\mathrm{x}$-ray tube alimentation is one of the important one and here, three different voltages were examined which were 150,200 and $300 \mathrm{kV}$. In this study, a dry Yamazuna sand which has a wide range of grain size distribution shown in Fig. 3 was used in which the minimum particle size was $0.001 \mathrm{~mm}$ while its maximum was $10 \mathrm{~mm}$ with $\mathrm{D}_{50}$ of about $0.54 \mathrm{~mm}$. A specimen size was $50 \mathrm{~mm}$ in diameter and $100 \mathrm{~mm}$ in height. This sand with dry condition was prepared under the density of $1.63 \mathrm{t} / \mathrm{m}^{3}$ in the plastic mould.

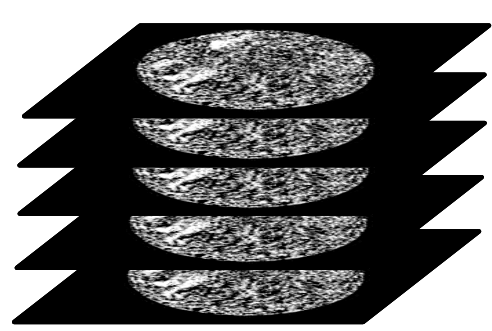

(a) A large number of cross sectionalimages

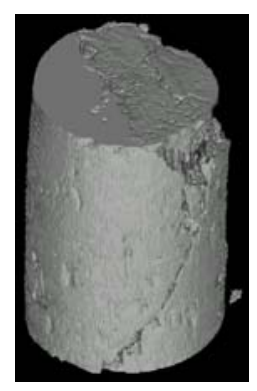

(b) Reconstructed 3D image

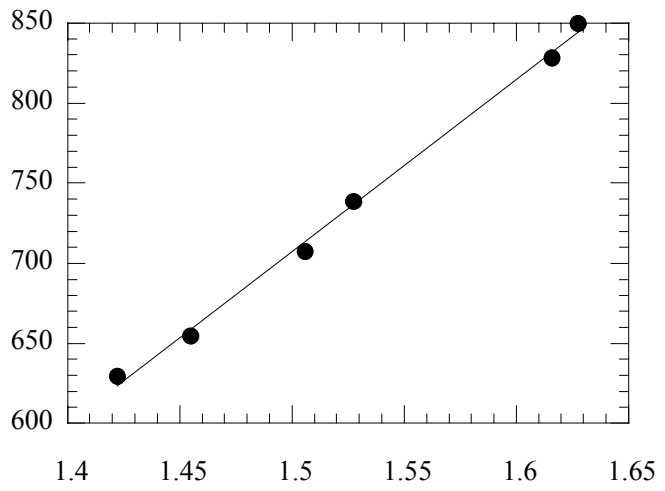

Figure 1. X-ray CT principle (Otani et al., 2000).

Figure 2. Relationship between CT-value and dry density of "Yamazuna sand". 
Fig. 4 shows the histograms of the obtained CT-values for all the three different voltage cases. As easily realized, each histogram is composed of two peaks; one representing the material of the specimen and the other one that of plastic mould and there is a wider range of CT-values for the case of lower voltage $(150 \mathrm{kV})$ compared to the other higher voltage cases. This result seems normal as we have more contrast with lower energy. The conclusion is that using the voltage of $150 \mathrm{kV}$ is better as the variation of the density in the materials wider. However, the x-ray beam with lower energy is more sensitive for beam hardening, which causes the edges of an object to appear brighter than the center even if the material is the same throughout. This phenomenon is often called "cupping effect" in X-ray Physics. Actually, since lower energy x-ray photons can be attenuated more easily than higher energy one, a polychromatic x-ray beam passing through an object loses the lower energy parts of its spectrum. This phenomenon is called "hardening" of the beam. There are a number of possible remedies for beam hardening, ranging from scanning preparation (hardware optimization) to data processing (software optimization). In this research, the former technique was examined for reducing beam hardening and here, a piece of cupper as an absorbing material in between the x-ray source and the specimen was placed as a filter. This objective is to absorb lower energy photons before $\mathrm{x}$-rays go through the specimen. Fig. 5 shows the distributions of CT-values along a diameter of the specimen for both of the cases of with and without filter for the same voltage condition. From this figure, there is a cupping effect on the distribution of the CT-values and a decrease of the cupping effect by using the filter of cupper is evident. Those contents have to be understood when the X-ray CT is used for geomaterials.

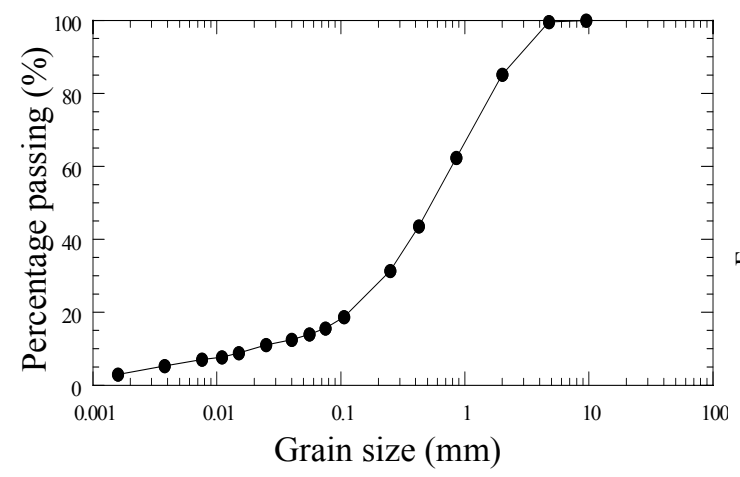

Figure 3. Grain size distribution in Yamazuna sand.

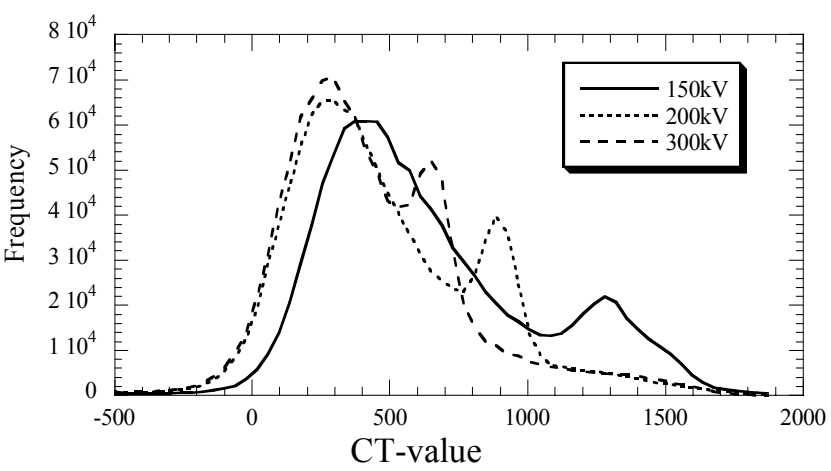

Figure 4. Voltage influence.

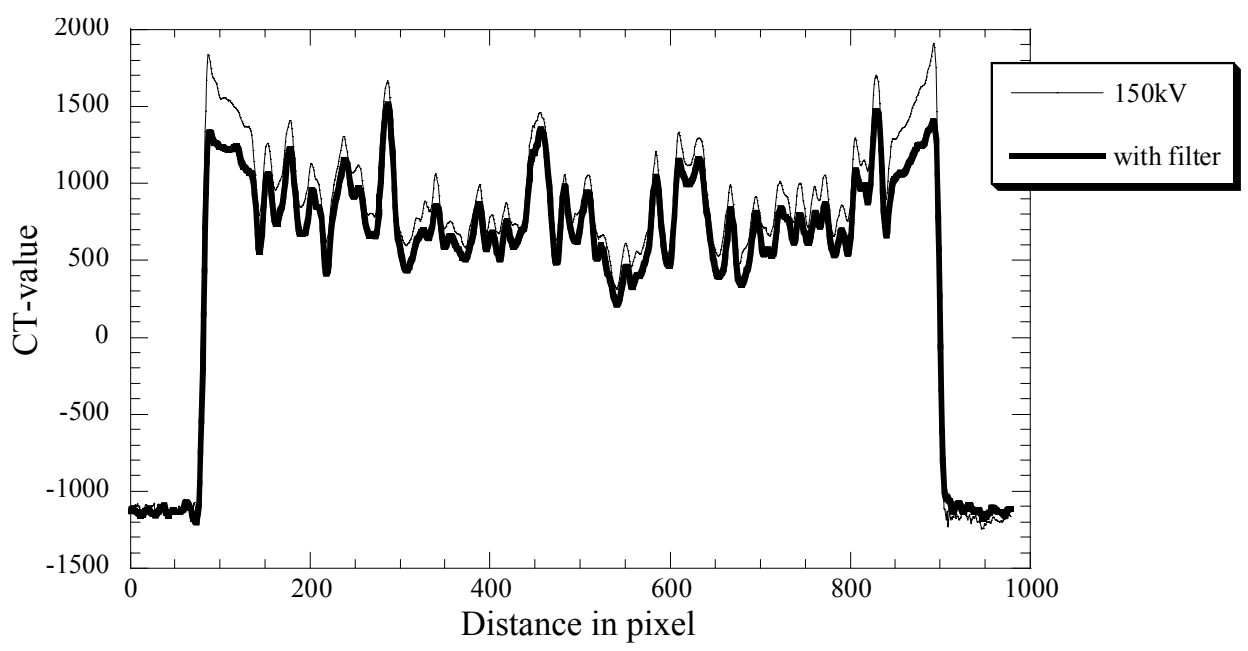

Figure 5. Beam hardening. 


\section{Measurements of displacements and strain [5]}

\subsection{Summary}

In-situ triaxial compression tests under drained condition were performed on Yamazuna sand at the X-Earth Center (Kumamoto University, Japan). The specimen was scanned during the test at different levels of loading in order to obtain full 3D images during the process of compression. The specimen size was $50 \mathrm{~mm}$ in diameter and $100 \mathrm{~mm}$ in height with a dry density of $1.63 \mathrm{t} / \mathrm{m}^{3}$ and $\mathrm{D}_{\mathrm{r}}=90 \%$. The axial loading rate was $0.3 \% / \mathrm{min}$ and confining pressures of $50 \mathrm{kPa}$ was applied in the test presented. For the CT scanning, the voltage used was $150 \mathrm{kV}$ and the electric current was $4 \mathrm{~mA}$ with cupper filter. The spatial resolution i.e. the size of the CT image voxels was $0.073 \times 0.073 \times 0.3 \mathrm{~mm}^{3}$. The specimen was scanned in continuous subsequent sections from bottom to top in order to obtain a 3D image of the whole specimen.

\subsection{Image analysis}

The DIC techniques have been used increasingly over the last 20 years in studies of the mechanics of a diverse range of materials, including recently geomaterials. DIC is essentially a mathematical tool for assessing the spatial transformation between images. The DIC analysis presented in this paper was carried out using the TomoWarp code developed at Laboratoire 3S-R, France. This code is a 3D (volumetric) DIC code providing a 3D volume of 3D displacement vectors between in-situ acquired CT images. Tomowarp follows the same basic steps as most DIC procedures and the strains based on the derived displacements under continuum assumption are calculated.

\subsection{Results and discussion}

Fig. 6 shows the force-displacement response from triaxial compression test presented here. The annotations on the curve (Initial, A, B, C and D) indicate the moments of the CT scans. First, there is a roughly linear increase that is followed by a curvature to the peak force at around $7 \%$. Then, the force decreases until the end of the test where there is the beginning of a plateau. Note that there are some stress relaxation phases due to stopping the loading during CT scanning. Most of the relaxation phase occurs almost immediately after the loading is stopped and not over the whole scanning period. Fig. 7 shows vertical slices of the middle part of the specimen at each scanning step. The images are presented in the top and bottom part of the figure respectively. In these grey scale images, black represents the air and white represents high-density material. In these images, the spatial variation in the soil density is clear and, due to the wide range of grain sizes and the given spatial resolution, the specimens appear to be a soil with large grains embedded in a matrix of fine grains (smaller than the resolution). Fig. 8 shows the distribution of the incremental shear strain at a vertical cross section resulted from DIC analysis. The section is roughly located at the same place as the vertical slices shown in Fig. 7. The white represents a strain greater than 0.3 and black represents no strain. This result clearly shows the evolution of the shear strain from two inclined shear bands before the stress peak into a single band after the peak. Moreover, it can be seen that the band starts as a wide, diffuse zone and converges to a narrow one in which the shear strain increases with an increase in axial loading. Fig. 9 shows the distribution of the incremental volumetric strain at the same vertical cross section position as in Fig. 7. The white represents a volumetric strain of -0.4 and less (i.e., dilation with the soil mechanics convention) and black represents a compressive strain greater than 0.4 (i.e., compression). The volumetric strain indicates dilation as the loading increases. Before the stress peak, in step A-B, two dilating bands have formed in the specimen. After step A-B, it is observed that zones of compression appear in the shear band. At step C-D, zones of dilation and compression exist through the whole of the band. 


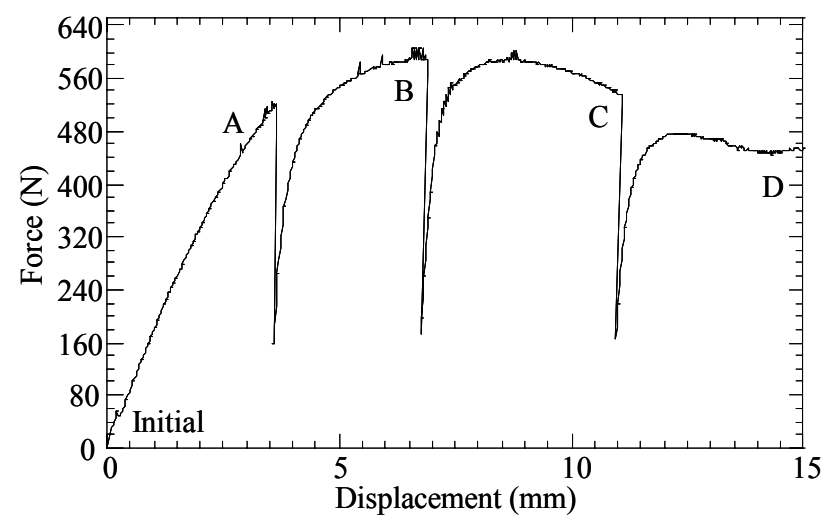

Figure 6. Force-displacement response.

Low

High density

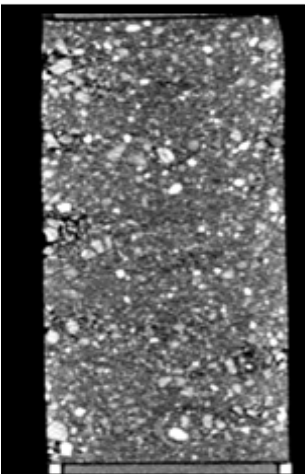

(a) Initial

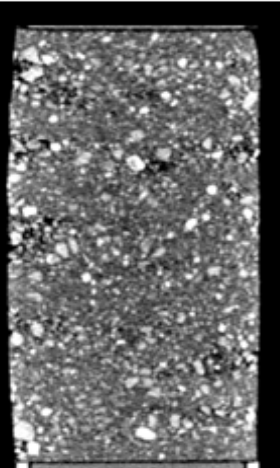

(b) Step A

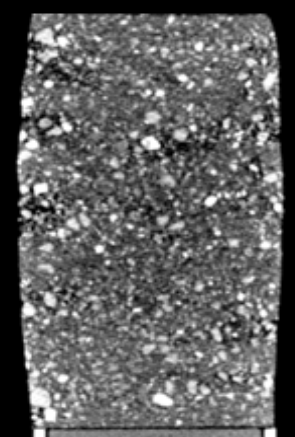

(c) Step B

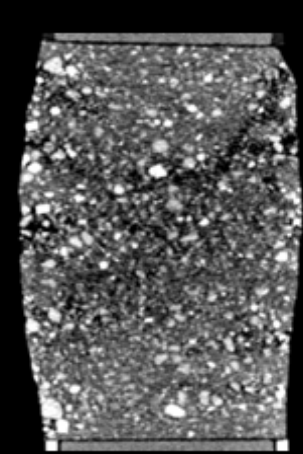

(d) Step C $50 \mathbf{~ k P a}$

Figure 7. Vertical CT slices through the loading for the $50 \mathrm{kPa}$ test (top) and the $150 \mathrm{kPa}$ (bottom).

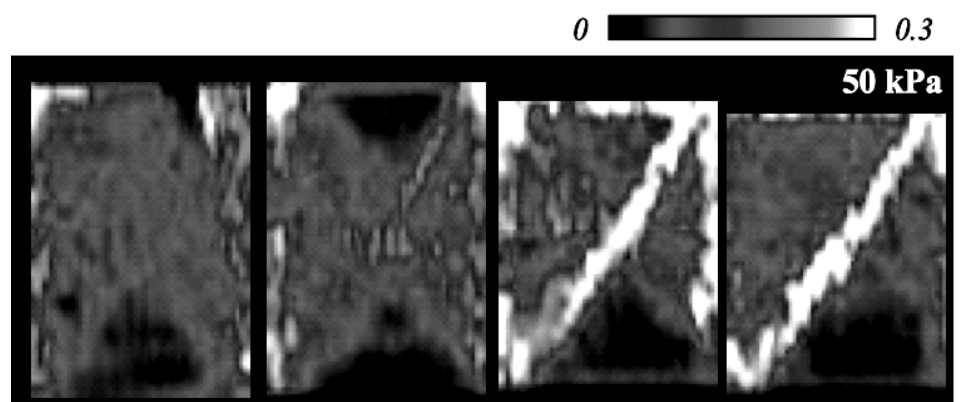

(a)Initial-A

(b) A-B

(c) $\mathrm{B}-\mathrm{C}$

(d) C-D

Figure 8. Distribution of incremental maximum shear strain from DIC.

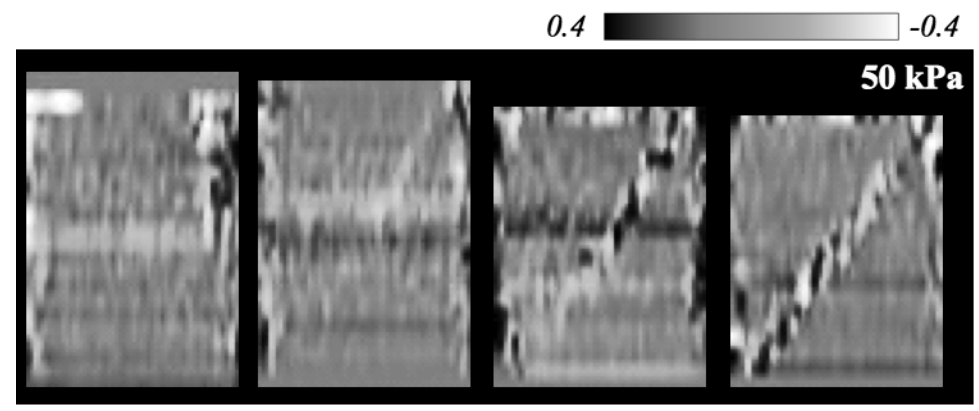

(a)Initial-A

(b) A-B

(c) $\mathrm{B}-\mathrm{C}$

(d) C-D

Figure 9. Distribution of incremental volumetric strain from DIC. 


\section{Trap-door problem [6]}

\subsection{Summary}

Trap-door problem has been investigated experimentally and theoretically by many authors. Most of these studies were performed in plane strain conditions (strip trap-door) or in axisymmetric conditions (circular trap-door). Even if observations on trap-door tests in plane strain conditions can be extended to non-plane strain conditions, it is important to notice that most of the arching phenomena occurring in real geotechnical earth structures correspond to non plane strain conditions. The manifestations of trap-door effect in real works are in most cases very local: sinkhole, tunnel face failure, and piled embankment. Consequently, experimental study of the trap-door problem in non-plane strain conditions is very important. The difficulty of observing the mechanisms occurring during trap-door tests performed in other conditions than plane strain conditions made that experimental trap-door problem in 3-dimensions were not investigated. The challenging point is to be able to observe the arching mechanisms despite the 3 dimensional testing conditions. The purpose of this study is to conduct experimental trap-door tests in the active mode and in 3-dimensions were conducted, with a square shaped trap-door. The use of the X-ray Computed Tomography (CT) technique makes possible the observation of the arching mechanisms occurring as the trap-door is moved downward, without altering the 3-dimensional aspect of the tests. Glass beads are used to compose the granular layers tested. Experimental results are compared to numerical results obtained with the Discrete Element Method (DEM). The exact conditions of the experimental tests are reproduced with the numerical model: model size, granular material characteristics and testing process.

\subsection{Test method}

\subsubsection{Test apparatus}

An acryl cylindrical container was used to contain the granular layers. This container, shown in Photo. 1 and in Fig.10 had an inside diameter of $240 \mathrm{~mm}$ and was supported by a horizontal steel plate under which the trap-door displacement device was placed. The trap-door hole $(40 \mathrm{mmx} 40 \mathrm{~mm})$ was created in the acryl bottom plate, placed inside the container and laying on the steel plate. The trapdoor itself (Fig.11) was an aluminium alloy plate of $40 \times 40 \mathrm{~mm}$ size in plan and $5 \mathrm{~mm}$ thickness. The value of the trap-door displacement $\delta$ was controlled with a gauge inserted between the two blocks. Considering $\delta=0 \mathrm{~mm}$ as the initial position of the trap-door, the trap-door was moved downward at once following different displacement steps: $\delta=0.1 \mathrm{~mm}, 1.0 \mathrm{~mm}, 2.0 \mathrm{~mm}, 5.0 \mathrm{~mm}, 10.0 \mathrm{~mm}$ and $20.0 \mathrm{~mm}$. Two kinds of experimental tests were distinguished: the firsts were dedicated to X-ray CT scanning, the seconds were dedicated to the measurement of the force on the trap-door in order to evaluate the load transfers occurring in the granular layer. The force applied by the granular material on the trapdoor was measured with one load cell located between the trap-door and its steel support in a centred position, which is shown in Fig.11. The force was measured for each displacement step $(\delta)$ and in order to evaluate the repeatability of the tests, 5 trap-door tests were made with the same testing conditions.

\subsubsection{Material}

The granular layers were composed on glass beads of diameter, $6 \pm 0.2 \mathrm{~mm}$. The density of these glass beads is $\rho_{\mathrm{g}}=2.52 \mathrm{~g} / \mathrm{cm}^{3}$. The minimal and maximal void ratios were measured as following: $\mathrm{e}_{\min }=0.562$ and $\mathrm{e}_{\max }=0.661$. Trap-door tests were performed on granular layers with a void ratio equal to $\mathrm{e}_{\min }$. The shear strength of the granular material is the main parameter influencing arching effect in trap-door tests, consequently peak and residual friction angles $\varphi_{\mathrm{p}}$ and $\varphi_{\mathrm{r}}$ of the glass beads were determined for the void ratio $e_{\min }$ corresponding to test density with triaxial compression test. The following friction angle values were obtained: $\varphi_{\mathrm{p}}=30^{\circ}$ and $\varphi_{\mathrm{r}}=24^{\circ}$. Before the trap-door test, the glass beads were placed in the container with successive layers of thickness, $20 \mathrm{~mm}$ each. The height of the glass beads sample was finally equal to $80 \mathrm{~mm}$. 


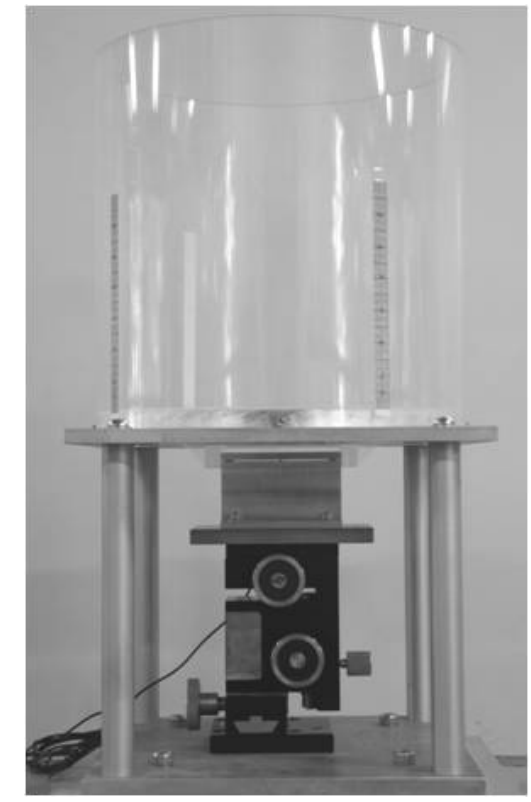

Photo 1. Picture of the trap-door apparatus

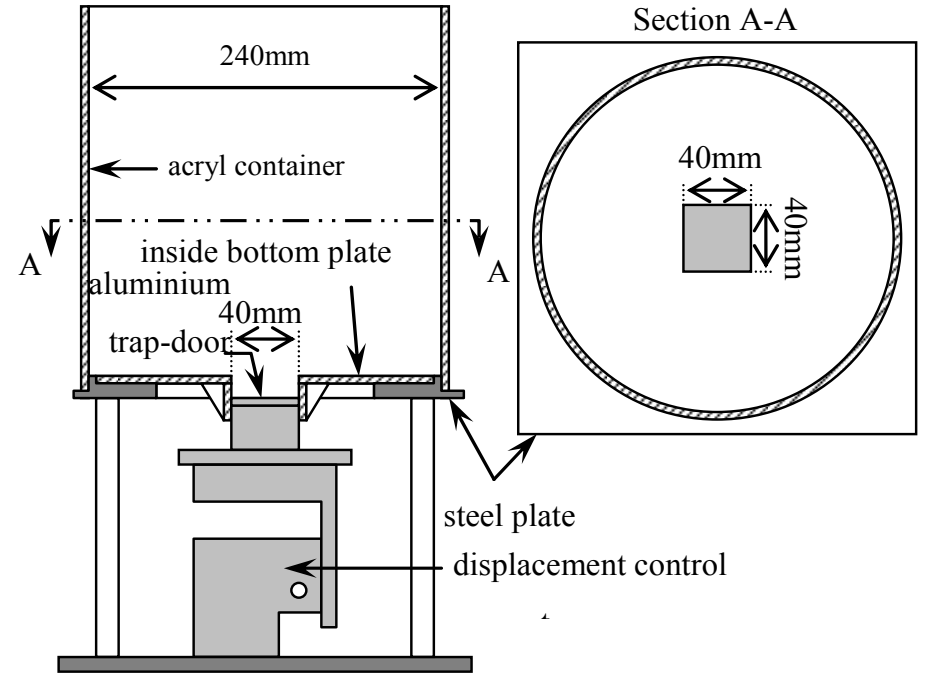

Figure 10. Configuration of the trap-door apparatus

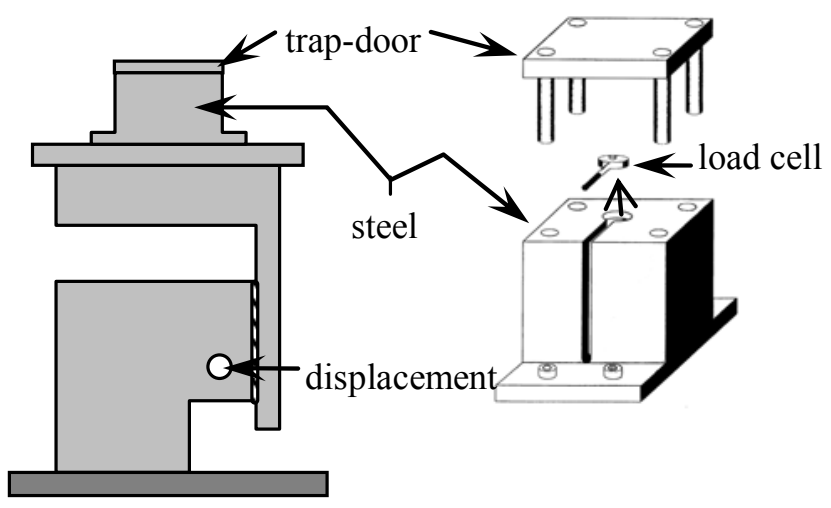

Figure 11. Configuration of the trap-door

\subsubsection{Discrete element method}

The numerical modelling of the trap-door problem was conducted with DEM based on the molecular dynamics. This method, used in 3-dimensions with a code called SDEC, considers the material as a collection of non-deformable bodies which interact with each other through their contact points. The law of the conservation of movement is applied on successive time-steps; in parallel interaction laws between particles allows reproducing a global behaviour of the collection of bodies which were spherical particles in the present study. More details of DEM analysis can be obtained form the reference [6]. 


\subsection{Results and discussion}

From now on, the volume or area of the granular material expanded due to trap-door displacement is called "expansion zone". The compared configurations between two different displacement steps of the trap-door are used to evaluate the propagation through the granular layer of the expansion zone due to trap-door displacement. Vertical cross section of samples in the median axis and the diagonal axis of the trap-door, as shown in Fig.12, are given for the different displacement steps of the trap-door in Fig.13 for experimental results and Fig.14 for numerical results. The cross sections showed a

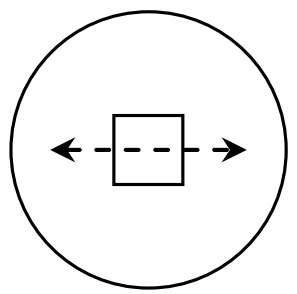

median cross

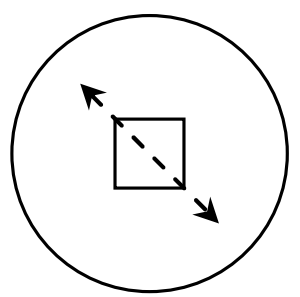

diagonal cross

Figure 12. Definition of the cross sections

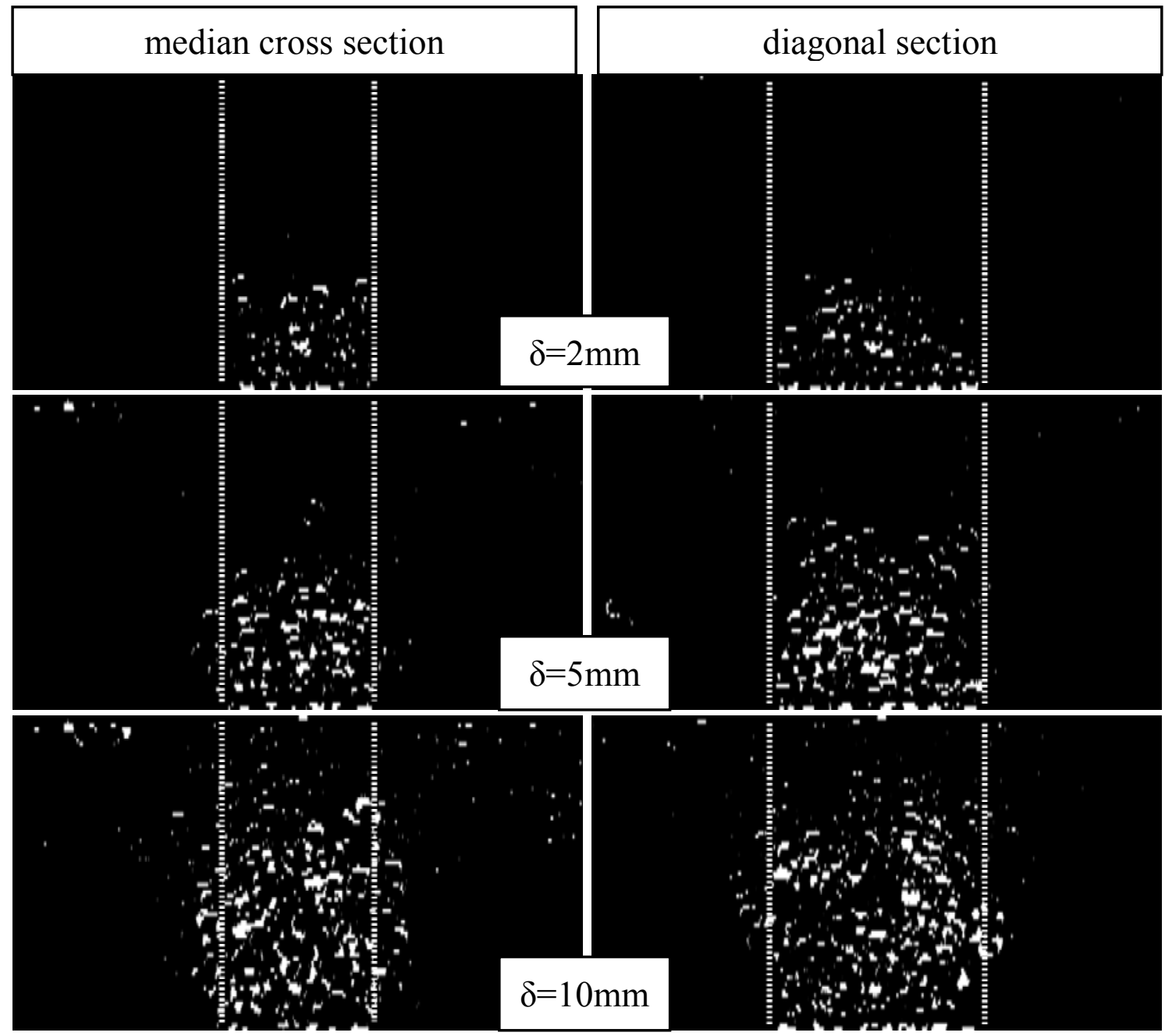

Figure 13. Vertical cross sections showing particle movements from CT scanning in which white color area corresponds to the area of particle movements in the median direction (left) and in the diagonal direction (right). 
progressive expansion of the area of particle movements. These areas obtained with experimental and numerical results are very similar. For large trap-door displacement $(\delta=10 \mathrm{~mm})$, it has to be noticed that in the median axis cross sections, the disturbed zone extends beyond the limit given by trap-door size while it remained contained in trap-door size limit in the diagonal axis. Further than plane views, a 3-dimensional reconstruction of the failure zone was made from the series of horizontal cross sections. The 3-dimensional views are displayed in Fig.15 and allowed to calculate the volume of the granular material affected by the trap-door displacement. The areas of particle movements extracted from experimental results and from numerical results are found to be very similar in shape but also in volume (see Fig.15). This good agreement can be obtained for all values of the trap-door displacement values $\delta=2,5$ and $10 \mathrm{~mm}$.

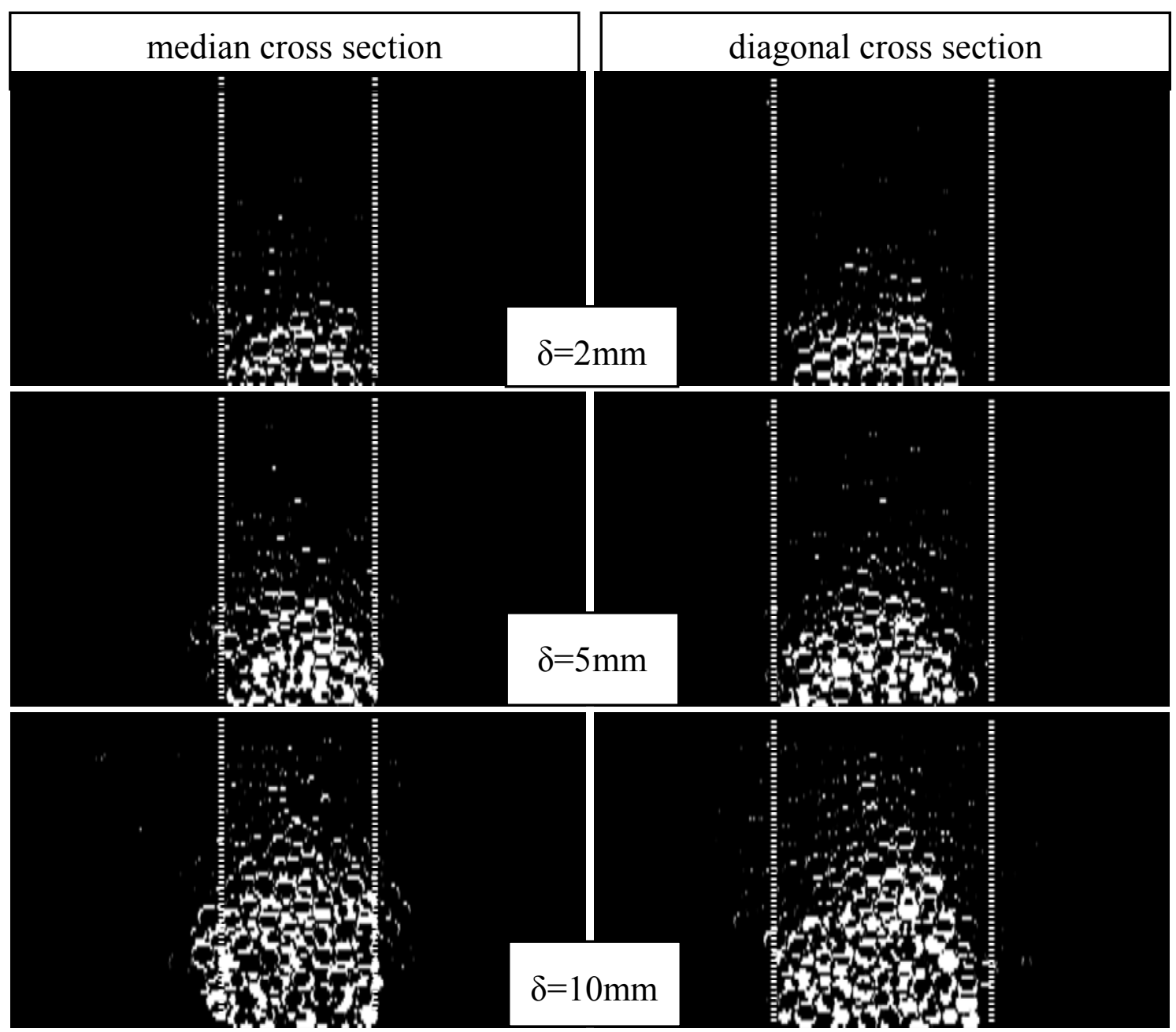

Figure 14. Vertical cross sections showing particle movements from DEM in which white color area corresponds to the area of particle movements in the median direction (left) and in the diagonal direction (right). 


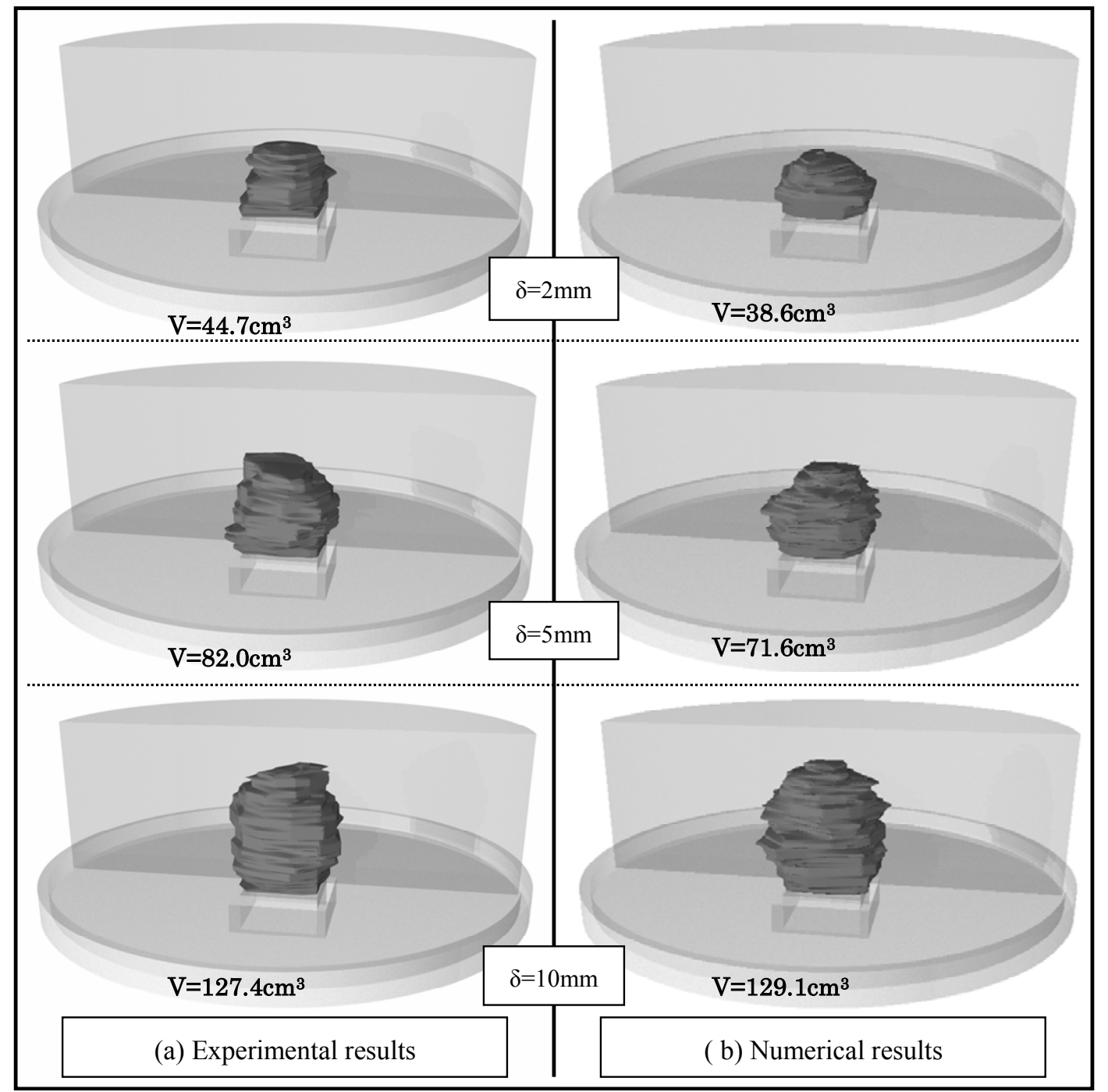

Figure 15. 3-dimensional view of the disturbed area for different displacement steps of the trap-door: experimental results (left) and numerical results (right)

\section{Conclusions}

Fundamental issues on the use of X-ray CT to geomaterials were discussed which were the contents of what you have to know about the use of X-ray CT for geomaterials. And two of our recent studies were introduced, which were 1) the use of X-ray CT data to quantitative measurements such as deformation and strains and 2) comparison of X-ray CT results with numerical analysis. Based on those results, it is clarified that the X-ray CT is the useful tool in the field of geomechanics and geotechnical engineering.

\section{References}

[1] Otani, J., Mukunoki, T. and Obara, Y. 2000 Soils and Foundations, 40(2)

[2] Otani, J., Mukunoki, T. and Kikuchi, Y. 2002: Soils and Foundations, 42(3)

[3] Otani, J., Mukunoki, T. and Sugawara, K. 2005 Soils and Foundations, 45(1)

[4] Watanabe Y., Lenoir N., Hall, S. and Otani, J. 2010 Proc. GeoX2010, New Orleans, U.S.A. (in print)

[5] Watanabe Y., Lenoir N., Otani J. and Nakai, T. 2010 Soils and Foundations (submitted)

[6] Chevalier B. and Otani, J. 2010 Soils and Foundations (submitted) 